\title{
Searching for the Bacterial Effector: The Example of the Multi-Skilled Commensal Bacterium Faecalibacterium prausnitzii
}

\author{
Rebeca Martín, Luis G. Bermúdez-Humarán and Philippe Langella* \\ National Institute of Agricultural Research, Commensals and Probiotics-Host Interactions Laboratory, Micalis Institute, \\ AgroParisTech, Paris-Sud University, Jouy-en-Josas, France
}

Faecalibacterium prausnitzii represents approximately $5 \%$ of the total fecal microbiota in healthy adults being one of the most abundant bacterium in the human intestinal microbiota of healthy adults. Furthermore, this bacterium has been proposed to be a sensor and a major actor of the human intestinal health because of its importance in the gut ecosystem. In this context, F. prausnitzii population levels have been found to be reduced in patients suffering from several syndromes and diseases such as inflammatory bowel diseases. These diseases are characterized by a breakage of the intestinal homeostasis called dysbiosis and the use of $F$. prausnitzii as a next generation probiotic (also called live biotherapeutics) has been proposed as a natural tool to restore such dysbiosis within the gut. Nevertheless, despite the potential importance of this bacterium in human health, little is known about its main effectors underlying its beneficial effects. In this perspective note, we aim to present the actual state in the research about $F$. prausnitzii effectors and the future milestones in this field.

Keywords: probiotic, commensal, Faecalibacterium, bacterial effectors, live-biotherapeutics

\section{INTRODUCTION}

Correspondence:

Philippe Langella

philippe.langella@inra.fr

Specialty section:

This article was submitted to

Food Microbiology,

a section of the journal

Frontiers in Microbiology

Received: 24 October 2017

Accepted: 13 February 2018

Published: 06 March 2018

Citation:

Martín R, Bermúdez-Humarán LG

and Langella $P$ (2018) Searching

for the Bacterial Effector: The Example

of the Multi-Skilled Commensal

Bacterium Faecalibacterium

prausnitzii. Front. Microbiol. 9:346.

doi: 10.3389/fmicb.2018.00346

Nowadays, humans can be considered as "meta-organisms" composed of 10-fold more microorganisms than human cells (Neish, 2009), which means 150-fold more genes than the human genome itself (Qin et al., 2010; Bruls and Weissenbach, 2011). These microorganisms, named microbiota (and by extension all their genes as a whole, named microbiome) are different depending on the tissue considered. The human gastrointestinal tract (GIT) is one of the most complex ecosystems. The advances of molecular techniques have shown that the collective adult human GIT microbiota is composed of up to 1000-1150 bacterial species (Frank et al., 2007; Qin et al., 2010). The predominant species (46-58\%) are those with low GC-content being the clostridium group the most abundant (Zoetendal et al., 2002; Qin et al., 2010). As a consequence of the mutualism established between the host and its microbiota, the GIT micro-ecosystem is key to maintain the homeostasis of a healthy individual (Leser and Molbak, 2009). Indeed, gut microbiota supplies essential nutrients, metabolize indigestible compounds and protects the host against colonization by opportunistic pathogens (Leser and Molbak, 2009; Martín et al., 2013). It also contributes to the development of the intestinal architecture as well as several immunomodulatory functions (Mazmanian et al., 2005). In some abnormal conditions, an imbalance in the microbial ecosystem may happen leading to a microbial imbalance known as dysbiosis. This dysbiosis is characterized by the growth of different non-predominant bacteria and/or the 
depletion of commensal ones that can lead to a situation of illness. As a result, this imbalance can also lead to the lack of some beneficial effects of these commensal bacteria, and thus unchain pathogenic conditions not only due to pathogen overgrowth (Martín et al., 2013).

\section{THE HUMAN GUT MICROBIOTA AS A SOURCE OF NEXT GENERATION PROBIOTICS (NGPS)}

As gut microbiota is now considered as a major actor underlying health, the idea of using some well-known microbiota components as next generation probiotics (NGPs) is very promising. Probiotics are "live microorganisms which when administered in adequate amounts confer a health benefit on the host" (FAO/WHO, 2001). Recently, this definition has been clarified by an expert panel of the International Scientific Association for Probiotics and Prebiotics (ISAPP) and re-defined as: "live microorganisms that, when administrated in adequate amounts, confer a health benefit on the host" (Hill et al., 2014). Most of the traditional probiotics belong to both lactic acid bacteria (LAB) and Bifidobacteria groups. These novel probiotics are thus considered as NGPs in contrast to traditional ones, which were not selected on the basis of human microbiota analysis. To consider a strain as probiotic, it should be: (i) well-characterized, (ii) achieve safety requirements, and (iii) confer beneficial effects on the host. However, a careful selection should be made in each case as probiotic properties are usually strain specific and cannot be extrapolated to another strain even belonging to the same species (Pineiro and Stanton, 2007; Gareau et al., 2010).

Similarly, as probiotic therapy is mainly based on restoring the normal balance of the intestinal ecosystem, we consider that the use of commensal bacteria as NGPs is a natural way to restore the dysbiotic situation within the GIT (Miquel et al., 2015a). Nevertheless, in contrast to most of probiotic lactobacilli or bifidobacteria strains, these NGPs are not listed neither on QPS (for Qualified Presumption of Safety), nor on GRAS (for Generally Recognized As Safe organisms) lists. Furthermore, as they have not a long record of safe consumption (precisely no documented safe use in Europe prior to 1997), these NGPs can only be used either as novel foods or drugs and the requirements to allow their market in Europe are more severe than for conventional probiotic strains (Miquel et al., 2015a). Nowadays, the inclusion of Faecalibacterium on the QPS list might be difficult due to its lack of a history of safe use. In addition, full toxicology assays and characterization of the strain are still needed for regulatory approval (Brodmann et al., 2017). We have reviewed this problematic in detail elsewhere (Miquel et al., 2015a).

\section{A FOCUS ON Faecalibacterium praustnizii}

As Faecalibacterium prausnitzii is an extremely oxygen sensitive (EOS) bacterium and difficult to grow (Duncan et al., 2002), most of the data about its physiology are based on metagenomic studies (Miquel et al., 2013), with some exceptions (Duncan et al., 2002; Ramirez-Farias et al., 2009; Lopez-Siles et al., 2012; Foditsch et al., 2014; Martín et al., 2017). F. prausnitzii is a member of the Clostridium group (phylum Firmicutes, class Clostridia, family Ruminococcaceae), specifically of the C. leptum group (Benevides et al., 2017), and represents around $5 \%$ of the total fecal microbiota in healthy adults being one of the most abundant bacterium in the human intestinal microbiota in healthy conditions (Hold et al., 2003). The first isolates were classified as Fusobacterium praustnizzi, but latter on its close relation with members of the C. leptum group was established thorough analysis of 16S rRNA gene (Miquel et al., 2014). The establishment of F. prausnitzii along the GIT may result from a combination of environmental factors such as other commensal species, redox mediators, oxygen concentration, mucus layer as well as bile salt concentrations and pH (Duncan et al., 2009; Lopez-Siles et al., 2012). In early infancy, F. prausnitzii abundance is very low and increases after the establishment of primo-colonizing bacteria (Hopkins et al., 2005). In the last years, this bacterium has been suggested as a biosensor and a major actor of the human intestinal health (Miquel et al., 2013). Indeed, F. prausnitzii levels have been found to be reduced in patients suffering from several syndromes and diseases such as inflammatory bowel diseases (IBDs), irritable bowel syndrome (IBS), colorectal cancer (CRC), obesity, and celiac disease (Balamurugan et al., 2008; Sokol et al., 2008; Neish, 2009; De Palma et al., 2010; Furet et al., 2010; Rajilic-Stojanovic et al., 2011) as well as in frail elderly (van Tongeren et al., 2005). We have more deeply review $F$. prausnitzii physiology and beneficial effect elsewhere (Miquel et al., 2013, 2014). Nevertheless, its EOS condition, make viable intestinal delivery one of the current challenges, due to their stringent survival conditions (El Hage et al., 2017).

Because of its important role in GIT homeostasis, F. prausnitzii is considered today as a potential NGPs. Its potential utilization has been already proposed for livestock animals, for instance the isolation and characterization of F. prausnitzii strains from stool of calves and piglets have been already performed (Foditsch et al., 2014). Also a specific formulation keeping this EOS bacterium alive at ambient air conditions has been also proposed for patients with intestinal dysbiosis-associated diseases (Khan et al., 2014). In order to evaluate its potential beneficial effects as a NGP, we have successfully used this bacterium in several murine models of IBD and IBS (Sokol et al., 2008; Martín et al., 2014a; Laval et al., 2015; Miquel et al., 2016) and other groups have also clearly demonstrated its beneficial effects in vivo (Carlsson et al., 2013; Rossi et al., 2015, 2016). Briefly, we have found that mice treated with either F. prausnitzii A2-165 or its supernatant (SN) present lower symptoms of inflammation in both acute and chronic chemically-induced colitis models as well as improved gut permeability and function in a model of gut impairment induced by dinitrobenzene sulfonic acid (DNBS) (Sokol et al., 2008; Martín et al., 2014a, 2015; Laval et al., 2015). We have also observed that A2-165 strain was able to reduce pain sensibility in 
partial restraint stress (PRS) and neo-maternal separation (NMS) murine models (Miquel et al., 2016). Furthermore, Carlsson et al. (2013) and Rossi et al. (2015, 2016) have found similar anti-inflammatory results as well as restoration of increased intestinal permeability in dextran sulfate sodium (DSS) induced colitis.

Staring the host pathways involved in the beneficial effects displayed by F. prausnitzii, in vitro tests have shown that: (i) although $F$. prausnitzii itself had no effect on IL-1 $\beta$-induced $\mathrm{NF}-\kappa \mathrm{B}$ activity, its SN abolished it in Caco- 2 cells transfected with a reporter gene for NF- $\kappa \mathrm{B}$ activity and (ii) peripheral blood mononuclear cell (PBMC) stimulation by F. prausnitzii led to significantly lower IL-12 and IFN- $\gamma$ production levels and higher secretion of IL-10 (Sokol et al., 2008). In this sense, human dendritic cells (DCs) stimulation with A2-165 and HTF-F strains also induced the production of IL-10 (Rossi et al., 2015). IL-10 has been established to be an important immune-regulatory cytokine that successfully suppresses the exacerbated mucosal immune response associated with colonic inflammation (Schreiber et al., 2000). This increasing in IL-10 induced by F. praustnizii has also been observed in vivo (Sokol et al., 2008). Furthermore, Rossi et al. (2016) found that the strain of F. prausnitzii A2-165 has a strong capacity to induce IL-10 in human and murine DCs and influence the T-cell differentiation in vitro and in vivo. In this sense, F. prausnitzii is also able to increase lymphocyte $\mathrm{T}$ regulatory (Treg) population in vivo after a colonic chemical challenge (Martín et al., 2014a) and has been identified as the major inducer of a specific IL-10 secreting Treg subset named CD4CD8 $\alpha$ lymphocytes present in the human colonic lamina propria and blood which is deficient in IBD patients (Sarrabayrouse et al., 2014).

\section{Faecalibacterium praustnizii EFFECTORS: WHERE DO WE STAND?}

Since the first study about $F$. prausnitzii performed in our laboratory almost 10 years ago (Sokol et al., 2008), we have sought to identify the bacterial effectors underlying its beneficial effects. We have been focused on its $\mathrm{SN}$ which showed antiinflammatory properties in both in vivo and in vitro experiments (Sokol et al., 2008; Martín et al., 2013). Our main hypothesis was that $F$. prausnitzii can produce an anti-inflammatory soluble molecule. First, we tried to identify the chemical nature of the molecule by submitting the SN to several enzymatic and physical methods and as shown in Figures $\mathbf{1 A - C}$, none of them were able to suppress the anti-inflammatory "properties" from the SN pointing out the possible presence of more than one effector on F. praustnizii SN. Of course, this result can also support an important role of butyrate in F. prausnitzii effects, although in vivo and in vitro experiments point out for a more complex situation (see below). Nevertheless, we need to consider that the beneficial effects of $F$. prausnitzii might not be present only in its $\mathrm{SN}$. For instance, we have found that some beneficial effects, such as the anti-nociceptive one, are observed only when the animals where treated with the bacterium but not with its SN (Miquel et al., 2016).
Some of the putative bacterial effectors identified until now are presented in the Figure $\mathbf{2}$ and are described in the next paragraphs.

\section{Butyrate}

Typically, as F. prausnitzii is one of the most abundant butyrateproducing species, its beneficial effects have been first attributed to butyrate. Butyrate is a short chain fatty acid (SCFA) wellknown for its pleiotropic and beneficial effects in the GIT (Duncan et al., 2009; Macfarlane and Macfarlane, 2011) as well as its immune-modulatory properties in vitro (Bocker et al., 2003). Furthermore, it is involved in the cross-feeding between butyrate producer bacteria and Bifidobacterium sp. which favors the coexistence of bifidobacterial strains with other bifidobacteria and with butyrate-producing colon bacteria in the human colon with the consequent benefit in the human health (Riviere et al., 2016). Microbial butyrate is considered key for colonic health, as it is an energy source for epithelial cells and is able to modulate oxidative stress and inflammation (Hamer et al., 2008). However, its role remains controversial as its effects seem to be dose- and time-dependent (Martín et al., 2013). In this sense, it also has different effects depending of the cell line tested (Bocker et al., 2003). For instance, regarding cells from intestinal origin, butyrate was found to decrease IL- 8 secretion in Caco-2 and human intestinal primary epithelial cells (HIPECs) and to enhance IL-8 production in both HT-29/p and HT-29 MTX cells (Bocker et al., 2003). Furthermore, not all butyrate producing-bacteria have the same anti-inflammatory profile in vitro. For instance, in $6 \mathrm{~h}$ TNF- $\alpha$ stimulated HT-29 cells, the SN of Roseburia intestinalis, a butyrate producer, does not have anti-inflammatory properties while F. prausnitzii SN does (Figure 1D).

Although the effect of butyrate is clearly present, the antiinflammatory capacities of $F$. prausnitzii do not seem to be limited to butyrate only. In previous studies, we have shown that $F$. prausnitzii-mediated butyrate production is not the only beneficial bacterial effector linked to this species in colitis models (Sokol et al., 2008; Martín et al., 2013; Miquel et al., 2015b). For instance, we found that the increase of the presence of 4hydroxybutyric acid in the feces, colon and caecum of dixenic mice colonized with F. prausnitzii A2-165 and a strain of Escherichia coli compared to monoxenic mice colonized only by the strain of $E$. coli was not directly linked to health parameters in a rat model of acute trinitrobenzenic acid (TNBS)-induced colitis although the increase of production of butyrate has been also verified by gas liquid chromatography (Miquel et al., 2015b). These results support previous findings, where we found that butyrate did not protect mice from TNBS-induced colitis, in contrast to F. prausnitzii A2-165 SN (Sokol et al., 2008).

\section{Other Active Metabolites}

The use of a gnotobiotic model including F. prausnitzii A2-165 strain and E. coli allowed us, using a metabolomic approach, to identify several metabolites that, in contrast to 4-hydroxybutyric acid, are associated to the beneficial effect of $F$. prausnitzii in a TNBS-acute model in rats (Miquel et al., 2015b). These metabolites were the salicylic acid, shikimic acid and raffinose, 
A

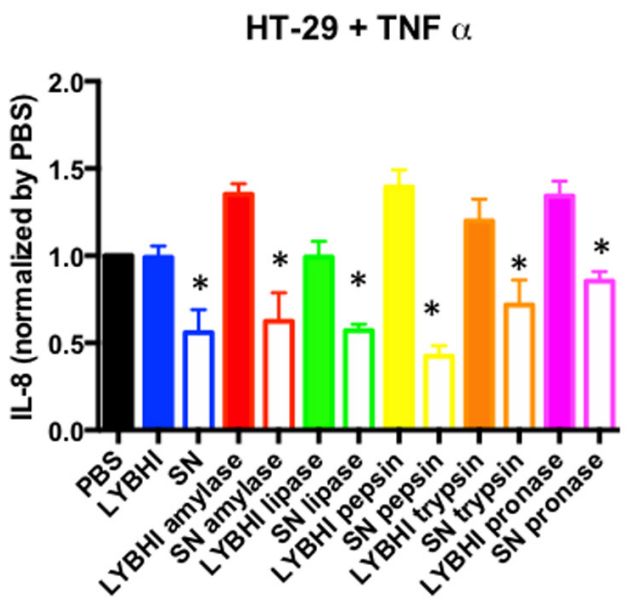

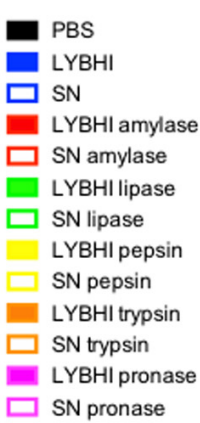

B

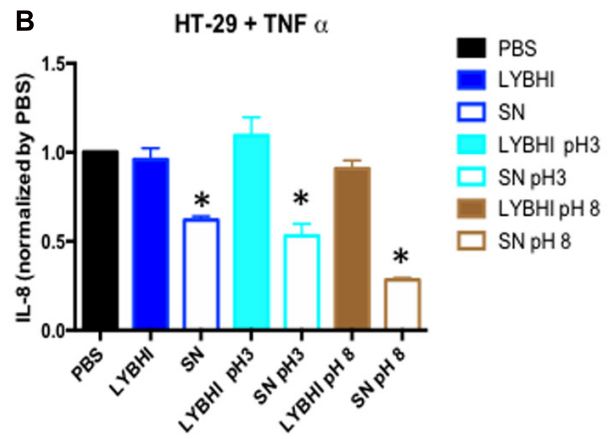

C

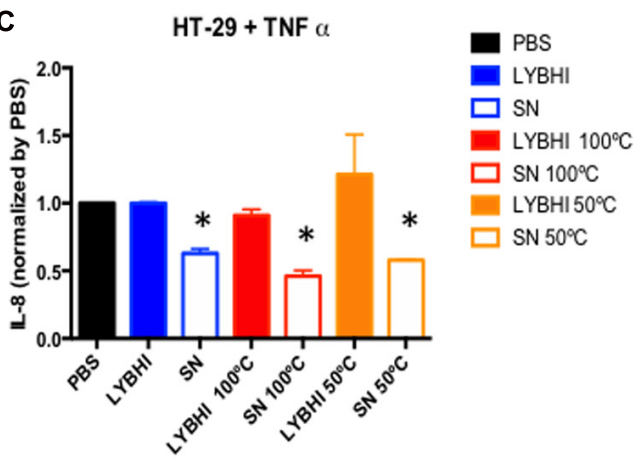

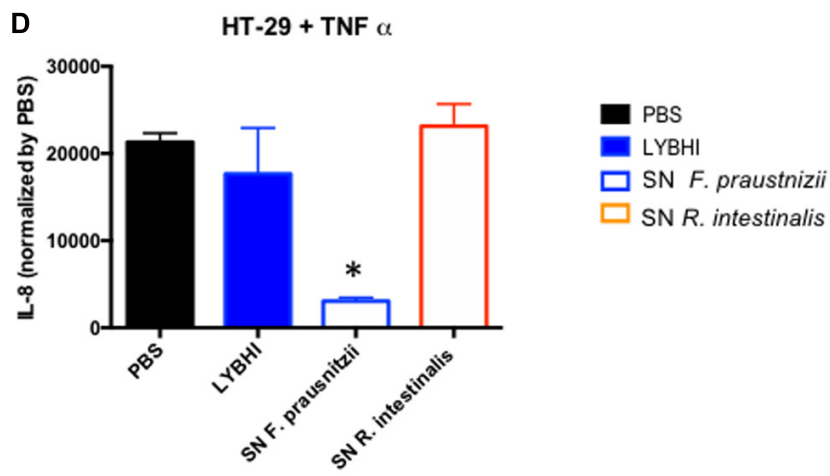

FIGURE 1 | In vitro immune-modulatory test on HT-29 cells stimulated with TNF- $\alpha$. Anti-inflammatory activity of Faecalibacterium prausnitzii A2-165 supernatant (SN) and culture medium (LYBHI) submitted to different treatments: (A) enzymatic treatments: amylase (red), lipase (green) pepsin (yellow), trypsin (orange) and pronase (pink), (B) pH modifications: $\mathrm{pH} 3$ (cyan) and pH 8 (brown), and (C) different temperatures: $100^{\circ} \mathrm{C}$ (red) and $50^{\circ} \mathrm{C}$ (orange). (D) Comparison of Roseburia intestinalis DSM14610T SN and F. prausnitzii A2-165 SN effect. Bacterial SNs were recovered, filtered and submitted to different treatments (if indicated) before being tested in co-incubation assays with HT-29 cells challenged with TNF- $\alpha$. IL-8 concentration was determined by ELISA and used as a read-out of the inflammatory status of the cells. The results were normalized by the negative control (PBS) (for material and methods details see Supplementary Data 1 ). ${ }^{*} p<0.05$ versus LYBHI (bacterial culture medium) $(n=3 * 3)$.

among others (Miquel et al., 2015b). Salicylic acid is used in the pharmaceutical industry to produce the amine derivate 5 -aminosalicylic acid (5-ASA or mesalamine) that it is nowadays used to treat patients suffering from IBD (Messori et al., 1994). Furthermore, shikimic acid is a precursor for the synthesis of several aromatic compounds among which we can also find the salicylic acid through the achorismate synthase pathway (Bochkov et al., 2012). These two compounds point out a key role of $F$. praustnizii in the biosynthesis of salicylic acid, precursor of 5-ASA, both anti-inflammatory molecules that should be linked 


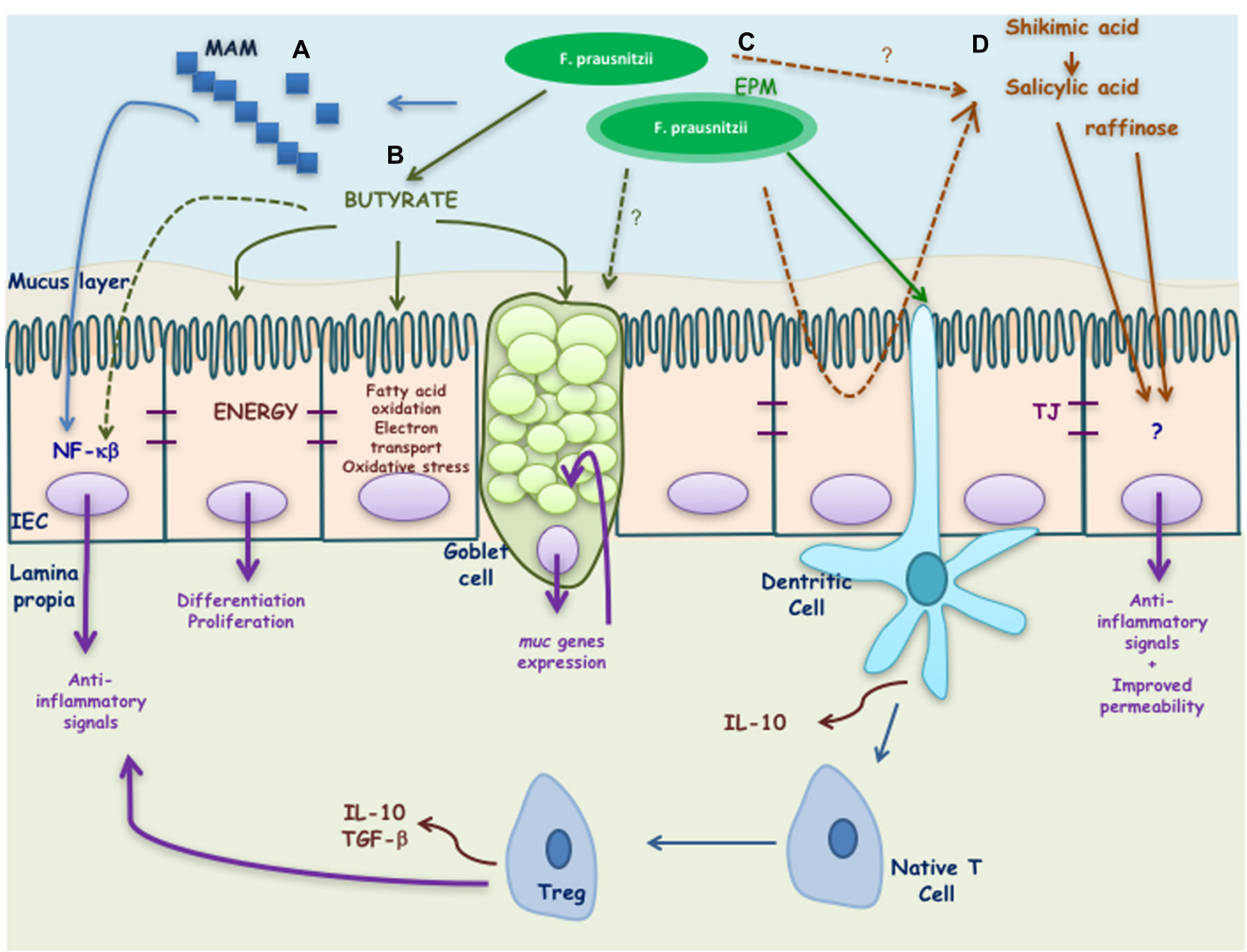

FIGURE 2 | Putative effectors of Faecalibacterium prausnitzii and its effects on the host. F. prausnitzii exerts its benefic effects by means of different effectors:

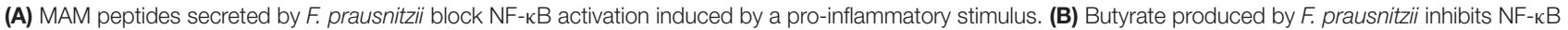
activation and interacts with the intestinal epithelial cells (IEC) driving to the activation of different genes involved on the differentiation, proliferation, and restitution of enterocytes. It is also involved on the regulation of fatty acid oxidation, electrons transport chain, oxidative stress, and apoptosis. In goblet cells it has been described to stimulate muc genes allowing a high production of mucus. (C) EPM produced by F. prausnitzii modulates IL-10 cytokine production in antigen presenting cells. Finally, (D) salicylic and shikimic acids are anti-inflammatory molecules able to block inflammation induced by a pro-inflammatory stimulus while raffinose is key in maintaining gut permeability.

to the in vivo anti-inflammatory effect observed in mice treated with $F$. praustnizii. In contrast, raffinose is an oligosaccharide only fermented by the gut microbiota that is not related to antiinflammatory effects, but with mucosal permeability, as raffinose permeation is key in maintaining gut permeability (Dawson et al., 1988). The raffinose could thus play a role in the improvement on gut permeability promoted by F. praustnizii (Carlsson et al., 2013; Laval et al., 2014). In this sense, we have found that F. praustnizii and its $\mathrm{SN}$ are able to counterbalance the increase in intestinal barrier permeability in a murine model of gut dysfunction induced by DNBS (Martín et al., 2015). Of note, in this simplified microbiota, we have identified metabolites that can be produced either by the host or by the bacteria, and the direct production of these compounds by F. prausnitzii has not yet been proved.

\section{Microbial Anti-inflammatory Molecule (MAM)}

Thanks to a peptidomic analysis using mass spectrometry of F. prausnitzii A2-165 strain SN, we have successfully identified seven peptides, all derived from the same anti-inflammatory molecule, a protein of $15 \mathrm{kDa}$ named MAM (ZP05614546.1) (Quevrain et al., 2016). Due to the difficulties to test directly the peptides or the protein, mainly due to solubility problems, indirect strategies were performed to determine their biological effect. Transfection of MAM cDNA in epithelial cells led to a significant decrease in the activation of the nuclear factor (NF)- $\mathrm{kB}$ pathway with a dose-dependent effect (Quevrain et al., 2016). This inactivation of NF- $\kappa B$ pathway was also observed in vivo using a transgenic model of mice producing luciferase under the control of NF- $\mathrm{KB}$ promoter (Breyner et al., 2017). Finally, the administration of a food-grade bacterium, Lactococcus lactis, delivering a plasmid encoding MAM was able to alleviate DNBS and DSS induced colitis in mice (Quevrain et al., 2016; Breyner et al., 2017). Although these promising results point out for the strong role of MAM on F. prausnitzii SN effect, the persistence of the anti-inflammatory effect of the SN after proteolytic treatment and the ability of F. prausnitzii $\mathrm{SN}$ to block other inflammatory pathways different from NF-kB (Martin et al., 2014b) point that MAM is not the 
only bacterial effector mediating $F$. praustnizii SN beneficial effects.

\section{Extracellular Polymeric Matrix (EPM)}

Rossi et al. (2015) found that the biofilm-forming strain F. praustnizii HTF-F was able to attenuate the clinical symptoms of DSS-induced colitis in a stronger manner than A2-165 strain. Furthermore, the intra-rectal administration of purified extracellular polymeric matrix (EPM) decrease the disease index of the mice indicating that it contributes strongly to the protective effects of HTF-F strain. However, although the authors concluded that the anti-inflammatory effects of $F$. prausnitzii HTF-F strain may be in part be due to the immune-regulating properties of the EPM, they were not able to rule out other possible strain differences such as colonization ability, stress resistance or in vivo fitness, among others. In fact, these parameters might also contribute to the efficacy of the strain as improved survival might impact on butyrate or MAM production for instance.

\section{FUTURE PERSPECTIVES}

The lack of clearness about F. prausnitzii effectors is linked to the lack of knowledge in its biology and phylogeny. Furthermore, as we mentioned above, probiotic characteristics are strainspecific, and therefore individual studies should be performed in order to determine the individual beneficial effects as well as the individual effectors linked to these effects. If we compare to a classic probiotic group, lactobacilli, we can find that although all lactobacilli are able to produce lactic acid (proved to have beneficial effects in some ecosystems such as the vaginal), not all of them are equipped with the same arsenal of bacterial effectors (bacteriocins, biosulfactants, $\mathrm{H}_{2} \mathrm{O}_{2}$, etc...) that are strain specific. In the case of Faecalibacterium, the framework should be similar, as even if all the strains are able to produce butyrate, other possible molecules could be responsible of additional strain-specific beneficial effects.

Recently, we have isolated a collection of novel F. prausnitzii strains form healthy volunteers that we have characterized (Miquel et al., 2015a) and analyzed for anti-inflammatory properties with the aim of selecting new NGPs candidates (Martín et al., 2017). The deeper phylogenic analysis of the complete genome of this bacterial collection joint to the genomes already present in the public data bases has revealed that there are at least three separate clusters, spanning the classical Phylogroups I and II already found in F. praustnizii (Lopez-Siles et al., 2012, 2016; Martín et al., 2017) and that some strains appear to represent a deeper, more divergent branch of the "Faecalibacterium prausnitzii" taxon (Benevides et al., 2017). This new truth about $F$. prausnitzii provides evidence that the phylogeny of F. praustnizii should be reconsidered. In line with these results, in the future we should take into account the presence of at least three different genospecies inside Faecalibacterium group to better characterize their beneficial effects, their crosstalk with the host and the possible effectors underlying these effects. Furthermore, the pangenome analysis have been performed to get a global view of the genome of this genus. A total of 10,366 core-genome codifying DNA sequences have been found (3.33-fold the average total number of genes of the 17 analyzed strains) (Benevides et al., 2017). Nevertheless, up today no extensive genomic description of this taxon has been finished, being an ongoing task with a key role in the future perspectives of the analysis of this genus.

\section{CONCLUDING REMARKS}

In this perspective article, we have tended to highlight the importance and problematic of asserting the probiotic characterization of a NGP, a field on the focus of the research of Frontiers in Microbiology audience. Nowadays, the research community recognizes the importance of $F$. prausnitzii in the human health. A decrease of this bacterium in the GIT has been linked to several diseases and syndromes but it is still not clear if this is a cause or a consequence of them. As mentioned above, this special condition, make this species a unique bacterial sensor and actor in the human health, mainly related with intestinal issues, but not only. Taking advantage of this, its use as NGP is being explored for both human and animal use. However, more research in its phylogeny, physiology, safety, and beneficial effects should be performed to fill the lack that currently exists between the knowledge of the biology of the bacterium and the medical interest that it produces. As an example, the analysis of the possible bacterial effectors taking into account the phylogeny and the biological effects related should be performed in more detail to find the best probiotic candidate and refine its use as biomarker in several human disorders.

\section{AUTHOR CONTRIBUTIONS}

$\mathrm{RM}, \mathrm{LB}-\mathrm{H}$, and $\mathrm{PL}$ designed the perspective and the experiments. RM wrote the manuscript and performed the experiments. LB-H and PL corrected the manuscript. All the authors approved the last version of the manuscript.

\section{ACKNOWLEDGMENTS}

RM receives a salary from Danone Nutricia Research in the framework of a postdoc contract.

\section{SUPPLEMENTARY MATERIAL}

The Supplementary Material for this article can be found online at: https://www.frontiersin.org/articles/10.3389/fmicb. 2018.00346/full\#supplementary-material 


\section{REFERENCES}

Balamurugan, R., Rajendiran, E., George, S., Samuel, G. V., and Ramakrishna, B. S. (2008). Real-time polymerase chain reaction quantification of specific butyrateproducing bacteria, Desulfovibrio and Enterococcus faecalis in the feces of patients with colorectal cancer. J. Gastroenterol. Hepatol. 23(8 Pt 1), 1298-1303. doi: 10.1111/j.1440-1746.2008.05490.x

Benevides, L., Burman, S., Martin, R., Robert, V., Thomas, M., Miquel, S., et al. (2017). New insights into the diversity of the genus Faecalibacterium. Front. Microbiol. 8:1790. doi: 10.3389/fmicb.2017.01790

Bochkov, D. V., Sysolyatin, S. V., Kalashnikov, A. I., and Surmacheva, I. A. (2012). Shikimic acid: review of its analytical, isolation, and purification techniques from plant and microbial sources. J. Chem. Biol. 5, 5-17. doi: 10.1007/s12154011-0064-8

Bocker, U., Nebe, T., Herweck, F., Holt, L., Panja, A., Jobin, C., et al. (2003). Butyrate modulates intestinal epithelial cell-mediated neutrophil migration. Clin. Exp. Immunol. 131, 53-60. doi: 10.1046/j.1365-2249.2003. 02056.x

Breyner, N. M., Michon, C., de Sousa, C. S., Vilas Boas, P. B., Chain, F., Azevedo, V. A., et al. (2017). Microbial anti-inflammatory molecule (MAM) from Faecalibacterium prausnitzii shows a protective effect on DNBS and DSSinduced colitis model in mice through inhibition of NF-kappaB pathway. Front. Microbiol. 8:114. doi: 10.3389/fmicb.2017.00114

Brodmann, T., Endo, A., Gueimonde, M., Vinderola, G., Kneifel, W., de Vos, W. M., et al. (2017). Safety of novel microbes for human consumption: practical examples of assessment in the European Union. Front. Microbiol. 8:1725. doi: $10.3389 /$ fmicb. 2017.01725

Bruls, T., and Weissenbach, J. (2011). The human metagenome: our other genome? Hum. Mol. Genet. 20, R142-R148. doi: 10.1093/hmg/ddr353

Carlsson, A. H., Yakymenko, O., Olivier, I., Hakansson, F., Postma, E., Keita, A. V., et al. (2013). Faecalibacterium prausnitzii supernatant improves intestinal barrier function in mice DSS colitis. Scand. J. Gastroenterol. 48, 1136-1144. doi: 10.3109/00365521.2013.828773

Dawson, D. J., Lobley, R. W., Burrows, P. C., Notman, J. A., Mahon, M., and Holmes, R. (1988). Changes in jejunal permeability and passive permeation of sugars in intestinal biopsies in coeliac disease and Crohn's disease. Clin Sci. 74, 427-431. doi: 10.1042/cs0740427

De Palma, G., Nadal, I., Medina, M., Donat, E., Ribes-Koninckx, C., Calabuig, M., et al. (2010). Intestinal dysbiosis and reduced immunoglobulin-coated bacteria associated with coeliac disease in children. BMC Microbiol. 10:63. doi: 10.1186/ 1471-2180-10-63

Duncan, S. H., Hold, G. L., Harmsen, H. J., Stewart, C. S., and Flint, H. J. (2002). Growth requirements and fermentation products of Fusobacterium prausnitzii, and a proposal to reclassify it as Faecalibacterium prausnitzii gen. nov., comb. nov. Int. J. Syst. Evol. Microbiol. 52(Pt 6), 2141-2146.

Duncan, S. H., Louis, P., Thomson, J. M., and Flint, H. J. (2009). The role of pH in determining the species composition of the human colonic microbiota. Environ. Microbiol. 11, 2112-2122. doi: 10.1111/j.1462-2920.2009.01931.x

El Hage, R., Hernandez-Sanabria, E., and Van de Wiele, T. (2017). Emerging trends in "smart probiotics": functional consideration for the development of novel health and industrial applications. Front. Microbiol. 8:1889. doi: 10.3389/fmicb. 2017.01889

FAO/WHO (2001). Expert Consultation on Evalutation of Health and Nutritional Properties of Probiotics in Food Including Powder Milk with Live Lactic Acid Bacteria. Available at: http://www.who.int/foodsafety/fs_management/en/ probiotic_guidelines.pdf

Foditsch, C., Santos, T. M., Teixeira, A. G., Pereira, R. V., Dias, J. M., Gaeta, N., et al. (2014). Isolation and characterization of Faecalibacterium prausnitzii from calves and piglets. PLoS One 9:e116465. doi: 10.1371/journal.pone.0116465

Frank, D. N., St Amand, A. L., Feldman, R. A., Boedeker, E. C., Harpaz, N., and Pace, N. R. (2007). Molecular-phylogenetic characterization of microbial community imbalances in human inflammatory bowel diseases. Proc. Natl. Acad. Sci. U.S.A. 104, 13780-13785. doi: 10.1073/pnas.070662 5104

Furet, J. P., Kong, L. C., Tap, J., Poitou, C., Basdevant, A., Bouillot, J. L., et al. (2010). Differential adaptation of human gut microbiota to bariatric surgery-induced weight loss: links with metabolic and low-grade inflammation markers. Diabetes Metab. Res. Rev. 59, 3049-3057. doi: 10.2337/db10-0253
Gareau, M. G., Sherman, P. M., and Walker, W. A. (2010). Probiotics and the gut microbiota in intestinal health and disease. Nat. Rev. Gastroenterol. Hepatol. 7, 503-514. doi: 10.1038/nrgastro.2010.117

Hamer, H. M., Jonkers, D., Venema, K., Vanhoutvin, S., Troost, F. J., and Brummer, R. J. (2008). Review article: the role of butyrate on colonic function. Aliment. Pharmacol. Ther. 27, 104-119. doi: 10.1111/j.1365-2036.2007.03562.x

Hill, C., Guarner, F., Reid, G., Gibson, G. R., Merenstein, D. J., Pot, B., et al. (2014). Expert consensus document. The international scientific association for probiotics and prebiotics consensus statement on the scope and appropriate use of the term probiotic. Nat. Rev. Gastroenterol. Hepatol. 11, 506-514. doi: $10.1038 /$ nrgastro. 2014.66

Hold, G. L., Schwiertz, A., Aminov, R. I., Blaut, M., and Flint, H. J. (2003). Oligonucleotide probes that detect quantitatively significant groups of butyrateproducing bacteria in human feces. Appl. Environ. Microbiol. 69, 4320-4324. doi: 10.1128/AEM.69.7.4320-4324.2003

Hopkins, M. J., Macfarlane, G. T., Furrie, E., Fite, A., and Macfarlane, S. (2005). Characterisation of intestinal bacteria in infant stools using real-time PCR and northern hybridisation analyses. FEMS Microbiol. Ecol. 54, 77-85. doi: 10.1016/ j.femsec.2005.03.001

Khan, M. T., van Dijl, J. M., and Harmsen, H. J. (2014). Antioxidants keep the potentially probiotic but highly oxygen-sensitive human gut bacterium Faecalibacterium prausnitzii alive at ambient air. PLoS One 9:e96097. doi: 10.1371/journal.pone.0096097

Laval, L., Martin, R., Natividad, J., Chain, F., Miquel, S., de Maredsous, C. D., et al. (2014). Lactobacillus rhamnosus CNCM I-3690 and the commensal bacterium Faecalibacterium prausnitzii A2-165 exhibit similar protective effects to induced barrier hyper-permeability in mice. Gut Microbes 6, 1-9. doi: 10.4161/19490976. 2014.990784

Laval, L., Martin, R., Natividad, J. N., Chain, F., Miquel, S., Desclee de Maredsous, C., et al. (2015). Lactobacillus rhamnosus CNCM I-3690 and the commensal bacterium Faecalibacterium prausnitzii A2-165 exhibit similar protective effects to induced barrier hyper-permeability in mice. Gut Microbes 6, 1-9. doi: 10.4161/19490976.2014.990784

Leser, T. D., and Molbak, L. (2009). Better living through microbial action: the benefits of the mammalian gastrointestinal microbiota on the host. Environ. Microbiol. 11, 2194-2206. doi: 10.1111/j.1462-2920.2009.01941.x

Lopez-Siles, M., Khan, T. M., Duncan, S. H., Harmsen, H. J., Garcia-Gil, L. J., and Flint, H. J. (2012). Cultured representatives of two major phylogroups of human colonic Faecalibacterium prausnitzii can utilize pectin, uronic acids, and host-derived substrates for growth. Appl. Environ. Microbiol. 78, 420-428. doi: 10.1128/AEM.06858-11

Lopez-Siles, M., Martinez-Medina, M., Suris-Valls, R., Aldeguer, X., Sabat-Mir, M., Duncan, S. H., et al. (2016). Changes in the abundance of Faecalibacterium prausnitzii phylogroups I and II in the intestinal mucosa of inflammatory Bowel disease and patients with colorectal cancer. Inflamm. Bowel Dis. 22, 28-41. doi: 10.1097/MIB.0000000000000590

Macfarlane, G. T., and Macfarlane, S. (2011). Fermentation in the human large intestine: its physiologic consequences and the potential contribution of prebiotics. J. Clin. Gastroenterol. 45(Suppl.), S120-S127. doi: 10.1097/MCG. ob013e31822fecfe

Martín, R., Chain, F., Miquel, S., Lu, J., Gratadoux, J. J., Sokol, H., et al. (2014a). The commensal bacterium Faecalibacterium prausnitzii is protective in DNBSinduced chronic moderate and severe colitis models. Inflamm. Bowel Dis. 20, 417-430. doi: 10.1097/01.MIB.0000440815.76627.64

Martin, R., Lenoir, M., Chain, F., Langella, P., and Bermudez-Humaran, L. G. (2014b). The dual role of MAPK pathway in the regulation of intestinal barrier: the role of the commensal bacterium Faecalibacterium prausnitzii on this regulation. Inflamm. Bowel Dis. 20, E17-E18. doi: 10.1097/MIB. 0000000000000070

Martín, R., Miquel, S., Benevides, L., Bridonneau, C., Robert, V., Hudault, S., et al. (2017). Functional characterization of novel Faecalibacterium prausnitzii strains isolated from healthy volunteers: a step forward in the use of F. prausnitzii as a next-generation probiotic. Front. Microbiol. 8:1226. doi: $10.3389 /$ fmicb.2017.01226

Martín, R., Miquel, S., Chain, F., Natividad, J. M., Jury, J., Lu, J., et al. (2015). Faecalibacterium prausnitzii prevents physiological damages in a chronic lowgrade inflammation murine model. BMC Microbiol. 15:67. doi: 10.1186/s12866015-0400-1 
Martín, R., Miquel, S., Ulmer, J., Kechaou, N., Langella, P., and BermudezHumaran, L. G. (2013). Role of commensal and probiotic bacteria in human health: a focus on inflammatory bowel disease. Microb Cell Fact. 12:71. doi: 10.1186/1475-2859-12-71

Mazmanian, S. K., Liu, C. H., Tzianabos, A. O., and Kasper, D. L. (2005). An immunomodulatory molecule of symbiotic bacteria directs maturation of the host immune system. Cell 122, 107-118. doi: 10.1016/j.cell.2005.05.007

Messori, A., Brignola, C., Trallori, G., Rampazzo, R., Bardazzi, G., Belloli, C., et al. (1994). Effectiveness of 5-aminosalicylic acid for maintaining remission in patients with Crohn's disease: a meta-analysis. Am. J. Gastroenterol. 89, 692-698.

Miquel, S., Beaumont, M., Martin, R., Langella, P., Braesco, V., and Thomas, M. (2015a). A proposed framework for an appropriate evaluation scheme for microorganisms as novel foods with a health claim in Europe. Microb Cell Fact. 14:48. doi: 10.1186/s12934-015-0229-1

Miquel, S., Leclerc, M., Martin, R., Chain, F., Lenoir, M., Raguideau, S., et al. (2015b). Identification of metabolic signatures linked to anti-inflammatory effects of Faecalibacterium prausnitzii. mBio 6:e00300-15. doi: 10.1128/mBio. 00300- 15

Miquel, S., Martin, R., Bridonneau, C., Robert, V., Sokol, H., Bermudez-Humaran, L. G., et al. (2014). Ecology and metabolism of the beneficial intestinal commensal bacterium Faecalibacterium prausnitzii. Gut Microbes 5, 146-151. doi: $10.4161 /$ gmic. 27651

Miquel, S., Martin, R., Lashermes, A., Gillet, M., Meleine, M., Gelot, A., et al. (2016). Anti-nociceptive effect of Faecalibacterium prausnitzii in non-inflammatory IBS-like models. Sci. Rep. 6:19399. doi: 10.1038/srep19399

Miquel, S., Martin, R., Rossi, O., Bermudez-Humaran, L., Chatel, J., Sokol, H., et al. (2013). Faecalibacterium prausnitzii and human intestinal health. Curr. Opin. Microbiol. 16, 255-261. doi: 10.1016/j.mib.2013.06.003

Neish, A. S. (2009). Microbes in gastrointestinal health and disease. Gastroenterology 136, 65-80. doi: 10.1053/j.gastro.2008.10.080

Pineiro, M., and Stanton, C. (2007). Probiotic bacteria: legislative frameworkrequirements to evidence basis. J. Nutr. 137(3 Suppl. 2), 850S-853S. doi: $10.1093 / \mathrm{jn} / 137.3 .850 \mathrm{~S}$

Qin, J., Li, R., Raes, J., Arumugam, M., Burgdorf, K. S., Manichanh, C., et al. (2010). A human gut microbial gene catalogue established by metagenomic sequencing. Nature 464, 59-65. doi: 10.1038/nature08821

Quevrain, E., Maubert, M. A., Michon, C., Chain, F., Marquant, J., Tailhades, S., et al. (2016). Identification of an anti-inflammatory protein from Faecalibacterium prausnitzii, a commensal bacterium deficient in Crohn's disease. Gut 65, 415-425. doi: 10.1136/gutjnl-2014-307649

Rajilic-Stojanovic, M., Biagi, E., Heilig, H. G., Kajander, K., Kekkonen, R. A., Tims, S., et al. (2011). Global and deep molecular analysis of microbiota signatures in fecal samples from patients with irritable bowel syndrome. Gastroenterology 141, 1792-1801. doi: 10.1053/j.gastro.2011.07.043

Ramirez-Farias, C., Slezak, K., Fuller, Z., Duncan, A., Holtrop, G., and Louis, P. (2009). Effect of inulin on the human gut microbiota: stimulation of Bifidobacterium adolescentis and Faecalibacterium prausnitzii. Br. J. Nutr. 101, 541-550. doi: 10.1017/S0007114508019880
Riviere, A., Selak, M., Lantin, D., Leroy, F., and De Vuyst, L. (2016). Bifidobacteria and butyrate-producing colon bacteria: importance and strategies for their stimulation in the human gut. Front. Microbiol. 7:979. doi: 10.3389/fmicb.2016. 00979

Rossi, O., Khan, M. T., Schwarzer, M., Hudcovic, T., Srutkova, D., Duncan, S. H., et al. (2015). Faecalibacterium prausnitzii strain HTF-F and its extracellular polymeric matrix attenuate clinical parameters in DSS-induced colitis. PLoS One 10:e0123013. doi: 10.1371/journal.pone.0123013

Rossi, O., van Berkel, L. A., Chain, F., Tanweer Khan, M., Taverne, N., Sokol, H., et al. (2016). Faecalibacterium prausnitzii A2-165 has a high capacity to induce IL-10 in human and murine dendritic cells and modulates T cell responses. Sci. Rep. 6:18507. doi: 10.1038/srep18507

Sarrabayrouse, G., Bossard, C., Chauvin, J. M., Jarry, A., Meurette, G., Quevrain, E., et al. (2014). CD4CD8alphaalpha lymphocytes, a novel human regulatory T cell subset induced by colonic bacteria and deficient in patients with inflammatory bowel disease. PLoS Biol. 12:e1001833. doi: 10.1371/journal.pbio.1001833

Schreiber, S., Fedorak, R. N., Nielsen, O. H., Wild, G., Williams, C. N., Nikolaus, S., et al. (2000). Safety and efficacy of recombinant human interleukin 10 in chronic active Crohn's disease. Crohn's disease IL-10 cooperative study group. Gastroenterology 119, 1461-1472. doi: 10.1053/gast.2000.20196

Sokol, H., Pigneur, B., Watterlot, L., Lakhdari, O., Bermudez-Humaran, L. G., Gratadoux, J. J., et al. (2008). Faecalibacterium prausnitzii is an antiinflammatory commensal bacterium identified by gut microbiota analysis of Crohn disease patients. Proc. Natl. Acad. Sci. U.S.A. 105, 16731-16736. doi: 10.1073/pnas.0804812105

van Tongeren, S. P., Slaets, J. P., Harmsen, H. J., and Welling, G. W. (2005). Fecal microbiota composition and frailty. Appl. Environ. Microbiol. 71, 6438-6442. doi: 10.1128/AEM.71.10.6438-6442.2005

Zoetendal, E. G., von Wright, A., Vilpponen-Salmela, T., Ben-Amor, K., Akkermans, A. D., and de Vos, W. M. (2002). Mucosa-associated bacteria in the human gastrointestinal tract are uniformly distributed along the colon and differ from the community recovered from feces. Appl. Environ. Microbiol. 68, 3401-3407. doi: 10.1128/AEM.68.7.3401-3407.2002

Conflict of Interest Statement: PL is one of the co-founders of NextBiotiX, a start-up aimed to produce an anti-inflammatory drug based on Faecalibacterium prausnitzii.

The other authors declare that the research was conducted in the absence of any commercial or financial relationships that could be construed as a potential conflict of interest.

Copyright (c) 2018 Martín, Bermúdez-Humarán and Langella. This is an open-access article distributed under the terms of the Creative Commons Attribution License (CC BY). The use, distribution or reproduction in other forums is permitted, provided the original author(s) and the copyright owner are credited and that the original publication in this journal is cited, in accordance with accepted academic practice. No use, distribution or reproduction is permitted which does not comply with these terms. 